\title{
Towards a Generalisation of Formal Concept Analysis for Data Mining purposes
}

\author{
Francisco J. Valverde-Albacete and Carmen Peláez-Moreno* \\ Dpto. de Teoría de la Señal y de las Comunicaciones. \\ Universidad Carlos III de Madrid \\ Avda. de la Universidad, 30. Leganés 28911. Spain \\ fva, carmen@tsc.uc3m.es
}

\begin{abstract}
In this paper we justify the need for a generalisation of Formal Concept Analysis for the purpose of data mining and begin the synthesis of such theory. For that purpose, we first review semirings and semimodules over semirings as the appropriate objects to use in abstracting the Boolean algebra and the notion of extents and intents, respectively. We later bring to bear powerful theorems developed in the field of linear algebra over idempotent semimodules to try to build a Fundamental Theorem for $\mathcal{K}$-Formal Concept Analysis, where $\mathcal{K}$ is a type of idempotent semiring. Finally, we try to put Formal Concept Analysis in new perspective by considering it as a concrete instance of the theory developed.
\end{abstract}

\section{Introduction and motivation}

When using Formal Concept Analysis for data mining purposes on non-binary data one is always forced to perform scaling procedures $[9,16]$ which carry a heuristic component sometimes difficult to justify in terms of the original data and requiring, in any case, a good deal of experience from the knowledge engineer.

From the point of view of the data it would be interesting to have alternative domains over which formal contexts could be defined and their lattices later built. There exist at least one such domains, the fuzzy domain tackled in [4,2]. Unfortunately, this domain presents operative problems when trying to build the concept lattices associated to them, mainly the fact that it is unclear whether the intuitions and tools developed in the standard case [9] can be translated to such undertaking. In particular, lattice building algorithms become much more demanding computationally speaking.

It would be much more interesting to develop an abstract theory of concept lattices sharing as many mathematical and algorithmic results and intuitions as

\footnotetext{
* This work has been partially supported by two grants for "Estancias de Tecnólogos Españoles en el International Computer Science Institute" of the Spanish Ministry of Industry for both authors and a Spanish Government CICYT grant TIC2002-03713 for the first author.
} 
possible with the concrete instance of Formal Concept Analysis, but somehow parameterized in the basic domain over which incidences could be defined.

For a proper generalisation, such an abstract theory should cater for the needs of Galois connection-induced concept lattices as well as the less understood adjunction-induced neighbourhood lattices [12]. Of course, such a theory should also encompass Boolean-defined incidences and their induced lattices, as we know them, as special cases, perhaps with outstanding or representative properties.

On the one hand, for the general enterprise of data mining any advance in this direction would be enlightening for a number of problems nowadays tackled with tools from conventional algebra. On the other hand, for the enterprise of coordinatising logical thought on the basis of an "algebra of concepts" as sketched in ([16], cfr. 4) this would broaden the range of tools at our disposal.

In a flight of fancy, let us suppose that we could have a "linear algebra" over extents and intents, that is, a conceptual "geometry". Then we could translate a wealth of methods and intuitions from "vector spaces" into Formal Concept Analysis with the appropriate caveats. For instance, if the polars could actually be represented by linear operators as matrices $I, I^{t}$ it would be feasible to solve problems like the following:

- Problem 1. find all the sets of objects closing to a particular intent, $B \in$ $2^{p \times 1} "$, that is, solve $I^{t} \cdot x=B$ for $x \in 2^{n \times 1}$.

Note that standard Formal Concept Analysis asserts that $I \cdot B$ is one such set, specifically its extent, but we might also be interested in describing the variety without resorting to the enumeration of all candidates, for example to apply the alternative recipe to reduce contexts of $[9](\S 1.5 .1 .2)$.

- Problem 2. given a set of objects $A_{1} \in 2^{n \times 1}$ find all other sets $A_{2} \in 2^{n \times 1}$ that map to its intent, that is, those such that $I^{t} \cdot A_{1}=I^{t} \cdot A_{2}$.

Note that this problem amounts to finding the quotient class of $A_{1}$ under $I^{t}$. In data mining terms this amounts to finding those input patterns that map to the same output pattern, i.e. classes of output-undistinguishable input patterns.

- Problem 3. find the quotient space of input patterns, $2^{n \times 1} / I^{t}$

This amounts to solving exhaustively problem 2 without finding first "representatives" like $A_{1}$ to guide it.

We could also think of dual problems involving the space of output patterns, produced by the transpose of the initial incidence.

The idea suggests itself that in this case the "geometric" properties may depend only secondarily on the Boolean domain and primarily on the "vector space" character of such sets. In which case, changing the domain in which sets and incidences take value would produce a flavour of "geometry", each different from the vanilla, standard Boolean flavour. Hence we put forward the following:

Hypothesis 1. Standard Formal Concept Analysis is a particular instance of $\mathcal{K}$-valued Formal Concept Analysis in which incidences and sets of objects and attributes take values in a suitable algebraic structure, $\mathcal{K}$. 
Consequently, in the rest of the paper we first introduce the mathematical notions leading to a suitable algebraic structure $\mathcal{K}$ and "modules over $\mathcal{K}$ " that can replace "modules over fields" (also "vector spaces") paying special attention to adjunctions and Galois connections between ordered sets. Later in section 3 we demonstrate a basic theorem for $\mathcal{K}$-valued Formal Concept Analysis and consider standard Formal Concept Analysis in as an instance of such construction. We conclude with a summary of contributions.

\section{Mathematical Preliminaries}

\subsection{Residuation Theory, Adjunctions and Galois Connections}

Lower semicontinous functions are (isotone) maps commuting with joins in partial orders, and upper semicontinuous functions are (isotone) maps commuting with meets in partial orders [1]. Given two partial orders $\langle P, \leq\rangle$ and $\langle Q, \leq\rangle$, we have:

- A map $f: P \rightarrow Q$ is residuated if inverse images of principal (order) ideals of $Q$ under $f$ are again principal ideals. Its residual map or simply residual, $f^{\#}: Q \rightarrow P$ is:

$$
f^{\#}(q)=\max \{p \in P \mid f(p) \leq q\}
$$

- A map $g: Q \rightarrow P$ is dually residuated if the inverse images of principal dual (order) ideals under $g$ are again dual ideals. Its dual residual map or simply dual residual, $g^{b}: P \rightarrow Q$ is:

$$
g^{b}(p)=\min \{q \in Q \mid p \leq g(q)\}
$$

Residuated maps are lower semicontinuous, while dually residuated maps are upper semicontinuous ([1], Th. 4. 50).

This abundance of concepts is fortunately simplified by a well-known theorem stating that residual maps are dually residuated, while dual residual maps are residuated, hence we may maintain only the two notions of residuated maps and their residuals. In fact, the two notions are so intertwined that we give a name to them: An adjoint pair of maps $(\lambda, \rho)$ is a pair $(\lambda: P \rightarrow Q, \rho: Q \rightarrow P)$ between two quasi ordered sets so that $\forall p \in P, q \in Q$,

$$
p \leq \rho(q) \Longleftrightarrow \lambda(p) \leq q \quad \text { equivalently } \quad p \leq \rho(\lambda(p)) \quad \& \quad \lambda(\rho(q)) \leq q
$$

If the order relation is partial the lower or left adjoint, $\lambda$ is uniquely determined by its right or upper adjoint, $\rho$, and conversely $([8], \S 1.1)$. The characterization theorem for adjoint maps $([8]$, p. 7$)$ states that $(\lambda, \rho)$ are adjoint if and only if, $\lambda$ is residuated with residual $\rho$, or equivalently, $\rho$ is dually residuated with $\lambda$ its dual residual. Hence adjunctions admit the following forms, using the following notation $(\lambda, \rho): P \leftrightharpoons Q$ to make the sets evident:

$$
(\lambda, \rho): P \leftrightharpoons Q \quad \Longleftrightarrow \quad\left(\lambda, \lambda^{\#}\right): P \leftrightharpoons Q \quad \Longleftrightarrow \quad\left(\rho^{b}, \rho\right): P \leftrightharpoons Q
$$


For Formal Concept Analysis, the more interesting notion of Galois connection, a contravariant pair of maps between the orders $P$ and $Q$, reads:

$$
p \leq \rho(q) \Longleftrightarrow q \leq \lambda(p) \quad \text { equivalently } \quad p \leq \rho(\lambda(p)) \quad \& \quad q \leq \lambda(\rho(q))
$$

A Galois connection can be equivalently described as an adjunction with the second order dualised:

$$
(\lambda, \rho): P \multimap Q \triangleq(\lambda, \rho): P \leftrightharpoons Q^{d}
$$

We introduce the diagram to the left of figure 1 as the pattern that carries the structures described in $([8], \S 1.2)$ :

- A closure system, $Q \rho$, the closure range of the right adjoint (see below).

- An interior system, $P \lambda$, the kernel range of the left adjoint (see below).

- A closure function (also "closure operator", $[12,6]) \gamma=\rho \circ \lambda$, from $P$ to the closure range $Q \rho$, with adjoint inclusion map $\hookrightarrow$.

- A kernel function (also "interior operator", [12]) $\kappa=\lambda \circ \rho$, from $Q$ to the kernel range $P \lambda$, with adjoint inclusion map $\hookrightarrow$.

However, due to the dualisation of the second set in Galois connections ranges are closures systems and both compositions closure operators (we write $\kappa^{*}$ for the new closure operator), resulting in the well-known dual isomorphism between closure ranges, illustrated to the right of figure 1 . Recall that a perfect adjunction
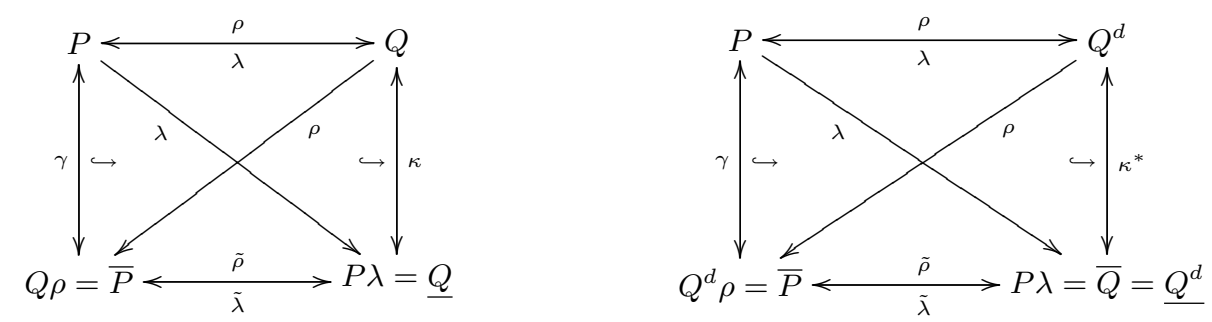

Fig. 1. Diagrams visually depicting the maps and structures involved in the adjunction $(\lambda, \rho): P \leftrightharpoons Q$ (left) and Galois connection $(\lambda, \rho) P \multimap Q$ (right) between two partially ordered sets (adapted from [8]).

is an adjunction where the diagram collapses to an order isomorphism between $P$ and $Q$, or equivalently, to an isomorphism between $\bar{P}$ and $Q$. Dually, for a perfect Galois connection, the diagram collapses to a single dual (antitone) order isomorphism between $\bar{P}$ and $\bar{Q}$. Prerequisites for this to happen are that the closure maps be identities, $\gamma(p)=p, \kappa^{*}(q)=q([8]$, p. 12).

\subsection{Idempotent Semirings}

This section aims at presenting the algebra that abstracts the features of the Boolean algebra which are adequate in our belief to generalise Formal Concept Analysis. 
A semiring $\mathcal{K}=\langle K, \oplus, \otimes, \epsilon, e\rangle$ is a structure where the additive structure, $\langle K, \oplus, \epsilon\rangle$, is a commutative monoid and the multiplicative one, $\langle K \backslash\{\epsilon\}, \otimes, e\rangle$, a monoid whose multiplication distributes over addition from right and left:

$$
\lambda \otimes(\mu \oplus \nu)=\lambda \otimes \mu \oplus \lambda \otimes \nu \quad(\mu \oplus \nu) \otimes \lambda=(\mu \otimes \lambda) \oplus(\nu \otimes \lambda)
$$

and whose neutral element is absorbing for $\otimes, \epsilon \otimes x=\epsilon, \forall x \in K$. On any semiring $\mathcal{K}$ left and right multiplications can be defined:

$$
\begin{array}{lrl}
L_{a}: K \rightarrow K & R_{a}: K \rightarrow K \\
b \mapsto L_{a}(b)=a b & b \mapsto R_{a}(b)=b a
\end{array}
$$

Hence a commutative semiring is a semiring whose multiplicative structure is commutative, and a semifield one whose multiplicative structure is a group. Thus, compared to a ring, a semiring crucially lacks additive inverses.

An idempotent semiring $\mathcal{K}$ is a semiring whose addition is idempotent:

$$
\forall a \in K, a \oplus a=a
$$

All idempotent commutative monoids $(K, \oplus, \epsilon)$ are endowed with a natural order $\forall a, b \in K, a \leq b \Longleftrightarrow a \oplus b=b$, which turns them into join-semilattices with least upper bound defined as $a \vee b=a \oplus b$. Moreover, for the additive structure of semiring $\mathcal{K}$ the neutral element is the infimum for this natural order, $\epsilon_{\mathcal{K}}=\perp$.

An idempotent semiring $\mathcal{K}$ is complete, if it is complete as a naturally ordered set and left $\left(L_{a}\right)$ and right $\left(R_{a}\right)$ multiplications are lower semicontinuous, that is, residuated.

Therefore, complete idempotent semirings, as sup-semilattices with infimum are automatically complete lattices $[6]$ with join $(\vee, \max$ or sup) and meet $(\wedge$, min or inf) ruled by the equations:

$$
\forall a, b \in K, a \leq b \Longleftrightarrow a \vee b=b \Longleftrightarrow a \wedge b=a
$$

Example 2. 1. The Boolean semiring $\mathcal{B}=\langle\mathbb{B}, \vee, \wedge, 0,1\rangle$, with $\mathbb{B}=\{0,1\}$, is complete, idempotent and commutative.

2. The completed Maxplus semiring $\bar{R}_{\max ,+}=\langle\mathbb{R} \cup\{ \pm \infty\}$, $\max ,+,-\infty, 0\rangle$, is a complete, idempotent semifield when defining $-\infty+\infty=-\infty$, that $\epsilon_{\mathcal{K}} \otimes \top_{\mathcal{K}}=\epsilon_{\mathcal{K}}$ for $\mathcal{K} \equiv \bar{R}_{\max ,+}$

3. The completed Minplus semiring $\bar{R}_{\text {min, }+}=\langle\mathbb{R} \cup\{ \pm \infty\}$, min, $+, \infty, 0\rangle$ is a complete, idempotent semifield with a similar completion to that of ex. 2 with $\infty+(-\infty)=\infty$, that is $\epsilon_{\mathcal{K}} \otimes \top_{\mathcal{K}}=\epsilon_{\mathcal{K}}$ for $\mathcal{K} \equiv \bar{R}_{\text {min },+}$.

The "values" populating a semiring are essentially positive or zero, hence we cannot expect to find "additive inverses" for them. The situation is less radical with multiplications in case the semiring exhibits the adequate order properties, as in idempotent semirings, because we may resort to residuation theory $[3,1]$ to try and invert such operations. But in that case we have the additional complexity of tracking the side of the multiplication, which applies 
particularly in case we want to "invert" the left and right multiplications of eqs. 1 , in which case the residuals are:

$$
\begin{array}{ll}
L_{a}^{\#}: K \rightarrow K & R_{b}^{\#}: K \rightarrow K \\
L_{a}^{\#}(c)=\vee\{\lambda \in K \mid a b \leq c\}=a \backslash c & R_{b}^{\#}(c)=\vee\{\lambda \in K \mid a b \leq c\}=c / b
\end{array}
$$

where the notation $a \backslash b$ reads "a under b" and $b / a$ reads "b over a", with:

$$
a b \leq c \Longleftrightarrow a \leq c / b \Longleftrightarrow b \leq a \backslash c
$$

Finally, note that if $\mathcal{K}$ is commutative, then $a \backslash b=b / a$.

Example 3. 1. For the boolean semiring, $a \backslash b=b / a=a \rightarrow b$, where $\rightarrow$ is the logical conditional, $a \mapsto 0 / a$ is the negation and $b \mapsto b / 1$ the identity.

2. For generic semiring $\mathcal{K}=\langle K, \oplus, \otimes, \epsilon, e\rangle$, the expression of a vector $x=$ $\left[x_{j}\right] \in K^{p \times 1}$ multiplying a matrix $R=\left[r_{i j}\right] \in K^{n \times p}$ is, $(R \cdot x)_{i}=\oplus_{j}\left(r_{i j} \otimes\right.$ $\left.x_{j}\right)$. Similarly, let $A, D \in K^{m \times n}, B \in K^{m \times p}$ and $C \in K^{n \times p}$, then the residuals for vectors and matrices over an idempotent semimodule may be obtained as ([1], p. 199):

$$
\begin{array}{rlrl}
C & =A \backslash B & D & =B / C \\
c_{i j} & =\bigwedge_{k=1}^{m} a_{k i} \backslash b_{k j} & d_{i j} & =\bigwedge_{k=1}^{p} b_{i k} / c_{j k}
\end{array}
$$

\subsection{Semimodules over Idempotent Semirings}

A semimodule over a semiring is defined in a similar way to a module over a ring, but allowances have to be made as to the sides of the multiplication ${ }^{1}$.

A left $\mathcal{K}$-semimodule $[14,13], \mathcal{X}=\left\langle X, \oplus, \epsilon_{X}\right\rangle$, is an additive commutative monoid endowed with a map $(\lambda, x) \mapsto \lambda \cdot x$ such that for all $\lambda, \mu \in K, x, z \in X$, and following the convention of dropping the symbol for the scalar action and multiplication for the semiring:

$$
\begin{aligned}
(\lambda \mu) x & =\lambda(\mu x) & & \epsilon_{K} x=\epsilon_{X} \\
\lambda(x \oplus z) & =\lambda x \oplus \lambda z & & e_{K} x=x
\end{aligned}
$$

The definition of a right $\mathcal{K}$-semimodule, $\mathcal{Y}$, follows the same pattern with the help of a right action, $(\lambda, y) \mapsto y \cdot \lambda$ and similar axioms to those of 5 . A $(\mathcal{K}, \mathcal{S})$-semimodule is a set $M$ endowed with left $\mathcal{K}$-semimodule and a right $\mathcal{S}$ semimodule structures, and a $(\mathcal{K}, \mathcal{S})$-bisemimodule a $(\mathcal{K}, \mathcal{S})$-semimodule such that the left and right multiplications commute. For a left $\mathcal{K}$-semimodule, $\mathcal{X}$, the left and right multiplications are defined as:

$$
\begin{array}{ll}
L_{\lambda}^{\mathcal{K}}: X \rightarrow X & R_{v}^{\mathcal{X}}: K \rightarrow X \\
x \mapsto L_{\lambda}^{\mathcal{K}}(x)=\lambda x & \lambda \mapsto R_{x}^{\mathcal{X}}(\lambda)=\lambda x
\end{array}
$$

\footnotetext{
${ }^{1}$ We are following essentially the notation of [5].
} 
And similarly, for a right $K$-semimodule. If $\mathcal{X}, \mathcal{Z}$ are left semimodules a morphism of left semimodules or left linear map $F: \mathcal{X} \rightarrow \mathcal{Z}$ is a map that preserves finite sums and commutes with the action: $F(\lambda v \oplus \mu w)=\lambda F(v) \oplus \mu F(w)$, and similarly, mutatis mutandis for right morphisms of right semimodules.

Idempotency and natural order in Semimodules. A $\mathcal{K}$-semimodule $\mathcal{M}$ over an idempotent semiring $\mathcal{K}$ inherits the idempotent law, $v \oplus v=v, \forall v \in M$, which induces a natural order on the semimodule by $v \leq w \Longleftrightarrow v \oplus w=$ $w, \forall v, w \in M$ whereby it becomes a sup-semilattice, with $\epsilon_{\mathcal{M}}$ the minimum.

When $\mathcal{K}$ is a complete idempotent semiring, a left $\mathcal{K}$-semimodule, $\mathcal{M}$ is complete if it is complete as a naturally ordered set and its left and right multiplications are (lower semi)continuous. Therefore, if $\mathcal{M}$ is complete for the natural order, it is also a complete lattice, with join and meet operations given by:

$$
v \leq w \Longleftrightarrow v \vee w=w \Longleftrightarrow v \wedge w=v
$$

All these definitions can be extended naturally to bisemimodules.

Example 4. 1. Each semiring, $\mathcal{K}$, is a left (right) semimodule over itself, with left (right) action the semiring product. Therefore, it is a $(\mathcal{K}, \mathcal{K})$ bisemimodule over itself, because both actions commute by associativity. Such is the case for the Boolean $(\mathcal{B}, \mathcal{B})$-bisemimodule, the Maxplus and the Minplus bisemimodules. These are all complete and idempotent.

2. The set of matrices $K^{n \times p}$ for finite $n$ and $p$ is a $\left(K^{n \times n}, K^{p \times p}\right)$-bisemimodule with matrix multiplication-like left and right actions and componentwise addition. The set of column vectors $K^{p \times 1}$ for finite $p$ is a $\left(K^{p \times p}, K\right)$-bisemimodule and the set of row vectors $K^{1 \times n}$ for finite $n$ a $\left(K, K^{n \times n}\right)$-bisemimodule with similarly defined operations. If $\mathcal{K}$ is idempotent (resp. complete), then all are idempotent (resp. complete) with the componentwise partial order their natural order.

Like in the semiring case, because of the natural order structure, the actions of idempotent semimodules also admit residuation: given a complete, idempotent left $\mathcal{K}$-semimodule, $\mathcal{X}$, we define for all $x, z \in X, \lambda \in K$ :

$$
\begin{array}{ll}
\left(L_{\lambda}^{\mathcal{K}}\right)^{\#}: X \rightarrow X & \left(L_{\lambda}^{\mathcal{K}}\right)^{\#}(z)=\bigvee\{x \in X \mid \lambda x \leq z\}=\lambda \backslash z \\
\left(R_{x}^{\mathcal{X}}\right)^{\#}: X \rightarrow K & \left(R_{x}^{\mathcal{X}}\right)^{\#}(z)=\bigvee\{\lambda \in K \mid \lambda x \leq z\}=z / x
\end{array}
$$

and likewise for a right semimodule, $\mathcal{Y}$.

There is a remarkable operation that changes the character of a semimodule while at the same time reversing its order by means of residuation: let $\mathcal{Y}$ be a complete, idempotent right $\mathcal{K}$-semimodule, its opposite semimodule is the complete left $\mathcal{K}$-semimodule $\mathcal{Y}^{o p}=\langle Y, \stackrel{o p}{\oplus, \stackrel{o p}{\rightarrow}}\rangle$ with the same underlying set $Y$, addition defined by $(x, y) \mapsto x \stackrel{o p}{\oplus} y=x \wedge y$ where the infimum is for the natural order of $\mathcal{Y}$, and left action:

$$
K \times Y \rightarrow Y \quad(\lambda, y) \mapsto \lambda \stackrel{o p}{\rightarrow} y=y / \lambda
$$


Consequently, the order of the opposite is the dual of the original order. For the opposite semimodule the residual definitions are:

$$
\begin{aligned}
& \lambda \bigwedge^{o p} x=\left(L_{\lambda}^{\mathcal{Y}^{o p}}\right)^{\#}(x)=\bigwedge\{y \in X \mid x \leq y / \lambda\}=x \cdot \lambda \\
& x \stackrel{o p}{/} y=\left(R_{y}^{\mathcal{Y}^{o p}}\right)^{\#}(x)=\bigvee\{\lambda \in K \mid x \leq y / \lambda\}=x \backslash y
\end{aligned}
$$

Note that we can define mutatis mutandis the opposite semimodule of a left $\mathcal{K}$-semimodule, $\mathcal{X}$, with right action $x \stackrel{o p}{\longleftarrow} \lambda=\lambda \backslash x$. Also, noticing that the first residual in eq. 8 is in fact an involution we may conclude that the operation of finding the opposite of a complete (left, right) $\mathcal{K}$-semimodule is an involution: $\left(\mathcal{M}^{o p}\right)^{o p}=\mathcal{M}$

Example 5 (Opposite Boolean semimodule). All semirings, $\mathcal{K}$, taken as $(\mathcal{K}, \mathcal{K})$-bisemimodules accept an opposite semiring, $\mathcal{K}^{o p}$. In particular, the op-

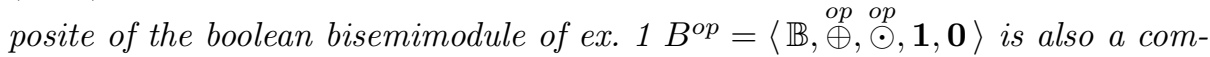
plete bisemimodule where addition is the min operation, notated by the meet $v \stackrel{o p}{\oplus} w=v \wedge w$. Consequently, its natural order is the inverse of the usual order for the lattice $\mathbf{2}$, the additively neutral element is $\epsilon_{B^{o p}}=\mathbf{1}$, which is the bottom for the opposite natural order, the unit is $e_{B^{\circ}}=\mathbf{0}$ and the action is the residual of the original action, $\lambda^{o p} \cdot x=\lambda \backslash x=x / \lambda$. In fact, the truth table for this connective is that of the logical conditional $\lambda \stackrel{o p}{\rightarrow} x=x \stackrel{o p}{\leftarrow} \lambda=\lambda \rightarrow x$.

Semimodules as vector spaces ${ }^{2}$. The elements of a semimodule are vectors. Given a semiring $\mathcal{K}$ and a left $\mathcal{K}$-semimodule $\mathcal{X}$, for each finite, nonvoid set $W \subseteq$ $X$, there exists an homomorphism $\alpha: K^{W} \rightarrow X, f \mapsto \bigoplus_{w \in W} f(w) w$. Moreover, $\alpha$ induces a congruence of semimodules $\equiv_{\alpha}$ on $K^{W}$, by $f \equiv_{\alpha} g \Longleftrightarrow \alpha(f)=$ $\alpha(g)$. Then $W$ is a set of generators or a generating family precisely when $\alpha$ is surjective, in which case any element $x \in X$ can be written as $x=\bigoplus_{w \in W} \lambda_{w} w$, and we will write $\mathcal{X}=\langle W\rangle$, that is, $\mathcal{X}$ is the span of $W$. A semimodule is finitely generated if it has a finite set of generators. For individual vectors, we say that $x \in W$ is dependent (in $W$ ) if $x=\bigoplus_{w \in W \backslash\{x\}} \lambda_{w} w$ otherwise, we say that it is free (in $W$ ). The set $W$ is linearly independent if and only if $\equiv_{\alpha}$ is the trivial congruence, that is, when $\bigoplus_{w \in W} f(w) w=\bigoplus_{w \in W} h(w) w \Longleftrightarrow f=h$, otherwise, $W$ is linearly dependent. Let $\operatorname{ker} \alpha=\left\{f \in K^{W} \mid \alpha(f)=0\right\}, W$ is weakly linearly independent if and only if $\operatorname{ker} \alpha=\{0\}$, otherwise it is weakly linearly dependent. A basis for $\mathcal{X}$ (over $\mathcal{K}$ ) is a linearly-independent set of generators, and a semimodule generated by a basis is free. By definition, in a free semimodule $\mathcal{X}$ with with basis $\left\{x_{i}\right\}_{i \in I}$ each element $x \in X$ can be uniquely written as $x=\bigoplus_{i \in I} \alpha_{i} x_{i}$, with $\left[a_{i}\right]_{i \in I}$ the coordinates of $x$ with respect to the basis. A weakly linearly-independent set of generators for $\mathcal{X}$ is a weak basis for $\mathcal{X}$ (over $\mathcal{K})$. The cardinality of a (weak) basis is the (weak) rank of the semimodule.

\footnotetext{
${ }^{2}$ Most of the material in this section is from [14], $\S 17$, and $[10,11,15]$.
} 
In such framework, notions in usual vector spaces have to be imported with care. For instance, the image of a linear map $F: \mathcal{X} \rightarrow \mathcal{Z}$ is simply the semimodule $\operatorname{Im} F=\{F(x) \mid x \in X\}$, but it is in general not free. Similarly, the following variant definition makes more sense: the (bi)kernel of the linear map $F: \mathcal{X} \rightarrow \mathcal{Z}$, is the congruence of semimodules $\operatorname{Ker} F=\left\{\left(x, x^{\prime}\right) \in X^{2} \mid F(x)=F\left(x^{\prime}\right)\right\}$.

Given a free semimodule $\mathcal{X}$ with basis $\left\{x_{i}\right\}_{i \in I}$, for each family $\left\{y_{i}\right\}_{i \in I}$ of elements of an arbitrary semimodule $\mathcal{Y}$ there is a unique morphism of semimodules $F: \mathcal{X} \rightarrow \mathcal{Y}$ such that $F\left(x_{i}\right)=y_{i}, \forall i \in I$, namely $F\left(\bigoplus_{i \in I} \lambda_{i} x_{i}\right)=\bigoplus_{i \in I} \lambda_{i} y_{i}$ and all the linear maps $\operatorname{Lin}(\mathcal{X}, \mathcal{Y})$ are obtained in this way([10], prop. $\S 73 ;[14]$, prop. $§ 17.12)$. That is, linear maps from free semimodules are characterized by the images of the elements of a basis.

On the other hand, a semiring $\mathcal{K}$ has the linear extension property if for all free, finitely generated $\mathcal{K}$-semimodules $\mathcal{X}, \mathcal{Y}$, for all finitely generated subsemimodules $\mathcal{Z} \subset X$ and for all $F \in \operatorname{Lin}(\mathcal{Z}, \mathcal{Y})$, there exists $H \in \operatorname{Lin}(\mathcal{X}, \mathcal{Y})$ such that $\forall x \in X, H(x)=F(x)$. The importance of this property derives from the fact that when the linear extension property holds, each linear map between finitely generated subsemimodules of free semimodules is represented by a matrix. In particular, when it holds for free, finitely generated (left) semimodules, $\mathcal{X}$ and $\mathcal{Y}$ with bases $\left\{x_{i}\right\}_{i \in I}$ and $\left\{y_{j}\right\}_{j \in J}$, each linear map is characterized by the $n \times p$-matrix $I=\left(F\left(x_{i}\right)_{j}\right)$, which sends vector $x$ with coordinates $x \simeq\left(\alpha_{i}\right)_{1 \leq i \leq n}$ to the vector $F(x) \simeq\left((x I)_{1}, \ldots,(x I)_{p}\right)$.

Idempotent vector spaces. Idempotent semimodules have additional properties which make them easier to work with as spaces. Therefore, when $\mathcal{K}$ is an idempotent semiring if a $\mathcal{K}$-semimodule has a (weak) basis, it is unique up to a rescaling map $y_{i}=\lambda x_{i}([15]$, th. $\S 3.1)$; and every finitely generated $\mathcal{K}$-semimodule has a weak basis $([15]$, cor. $\S 3.6)$.

Importantly, the linear property holds in every idempotent semiring which is a distributive lattice for the natural order $([10]$, th. $\S 83)$. This is the case for $\mathcal{K}=\mathbb{B}$, the boolean semiring and $\mathcal{K}=\bar{R}_{\text {max }}$. Therefore, in such semimodules, modulo a choice of bases for $\mathcal{X}$ and $\mathcal{Y}$, we may identify $\mathcal{X} \cong \mathcal{K}^{1 \times n}$ and $\mathcal{Y} \cong \mathcal{K}^{1 \times p}$, and linear maps to matrix transformations $\operatorname{Lin}(\mathcal{X}, \mathcal{Y}) \cong \mathcal{K}^{n \times p}, I: \mathcal{K}^{1 \times n} \rightarrow$ $\mathcal{K}^{1 \times p}, x \mapsto x I$. When passing from left to right semimodules this should read $\mathcal{K}^{p \times 1} \rightarrow \mathcal{K}^{n \times 1}, y \mapsto I y$

Constructing Galois connections in semimodules. Given a complete idempotent semiring $\mathcal{K}$, we call predual pair a complete left $\mathcal{K}$-semimodule $\mathcal{X}$ together with a complete right $\mathcal{K}$-semimodule $\mathcal{Y}$ equipped with a bracket $\langle\cdot \mid \cdot\rangle: X \times Y \rightarrow$ $Z$ to a complete $\mathcal{K}$-bisemimodule $\mathcal{Z}$, such that, for all $x \in X, y \in Y$ the maps:

$$
\begin{array}{ll}
R_{x}: Y \rightarrow Z & L_{y}: X \rightarrow Z \\
y \mapsto\langle x \mid y\rangle & x \mapsto\langle x \mid y\rangle
\end{array}
$$

are respectively left and right linear, and continuous. The most usual choice of bisemimodule $\mathcal{K}$ gives the bilinear forms, but one may also choose $\mathcal{K}^{o p}$. 
The following construction is due to Cohen et al. [5]: for a bracket $\langle\cdot \mid \cdot\rangle$ : $X \times Y \rightarrow Z$ and an arbitrary element $\varphi \in Z$, which we call the pivot, define the maps:

$$
\begin{array}{ll}
i_{l}: X \rightarrow Y & i_{l}(x)=L_{x}^{\#}(\varphi)=\bigvee\{y \in Y \mid\langle x \mid y\rangle \leq \varphi\} \\
i_{r}: Y \rightarrow X & i_{r}(y)=R_{y}^{\#}(\varphi)=\bigvee\{x \in X \mid\langle x \mid y\rangle \leq \varphi\}
\end{array}
$$

which may be shortened to: $i_{l}(x)=x^{-}$and $i_{r}(y)={ }^{-} y$. We have $\langle x \mid y\rangle \leq$ $\varphi \Longleftrightarrow y \leq x^{-} \Longleftrightarrow x \leq{ }^{-} y$, whence $i_{l}:(X, \leq) \rightarrow\left(Y, \leq^{d}\right)$ is residuated with residual $i_{l}^{\#}=i_{r}$ ([5], proof of prop. 24) hence, $\left(i_{l}, i_{r}\right)$ is an adjunction between $\mathcal{X}$ and $\mathcal{Y}^{o p}$ or equivalently, a Galois connection between $\mathcal{X}$ and $\mathcal{Y}$. Figure 2 depicts the morphisms and structures induced by such Galois connection. Note that the closure lattices $\bar{X}=\mathcal{Y} i_{r}$ and $\underline{Y}=\mathcal{X} i_{l}$ do not agree with their ambient vector spaces ${ }^{3}$ in their joins, but only in their meets!

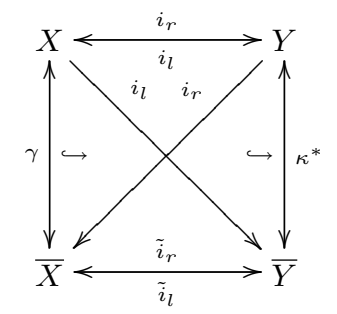

Fig. 2. The Galois connection $\left(i_{l}, i_{r}\right): \mathcal{X} \multimap \mathcal{Y}$ of the maps induced by $(\langle\cdot \mid \cdot\rangle, \varphi)$.

This construction is affected crucially by the choice of a suitable pivot $\varphi$ : in the operations of residuation only those pairs $(x, y) \in X \times Y$ are considered whose degree amounts to at most $\varphi$. Therefore we can think of the pivot as a maximum degree of existence allowed for the pairs.

Example 6. 1. (Involutions). The above construction can be used to obtain a family of different Galois connections between $\mathcal{X}_{m} \simeq \mathcal{K}^{m \times 1}$ and $\mathcal{Y}_{m} \simeq \mathcal{K}^{1 \times m}$ : define $\langle x \mid y\rangle=\bigoplus_{i} x(i) \otimes y(i)$, which is a predual pair for $\mathcal{Z}=\mathcal{K}$ ([5], ex. $\S 21)$, then $\psi_{m} \stackrel{\text { def }}{=}\left(i_{l}, i_{r}\right)_{m}: X_{m} \multimap Y_{m}$, as above, is a Galois Connection for each finite $m$.

2. (Galois connection between free row and column semimodules, [5] $\S 4.5$, adapted) Given a matrix ${ }^{4} R \in \mathcal{K}^{n \times p}$, the free complete semimodules $\mathcal{X}=\mathcal{K}^{1 \times n}$ and $\mathcal{Y}=\mathcal{K}^{p \times 1}$ form a predual pair for the bracket $\langle x \mid y\rangle_{R}=$

\footnotetext{
${ }^{3}$ Recall $\mathcal{X}$ and $\mathcal{Y}$ are both complete lattices as well as free vector spaces.

${ }^{4}$ Note that we are avoiding here giving using a generic $I$ for a relation because that name traditionally denotes unitary matrices.
} 
$x R y$. For such construction, define for a specific $\varphi \in K$ :

$$
\begin{aligned}
& x_{\varphi}^{-}=\bigvee\{y \mid x R y \leq \varphi\}=(x R) \backslash \varphi \\
& \bar{\varphi} y=\bigvee\{x \mid x R y \leq \varphi\}=\varphi /(R y)
\end{aligned}
$$

hence we have: $\bar{Y}=\left\{(x R) \backslash \varphi \mid x \in \mathcal{K}^{n \times 1}\right\}$ and $\bar{X}=\left\{\varphi /(R y) \mid y \in \mathcal{K}^{1 \times p}\right\}$ whence $\psi_{R} \stackrel{\text { def }}{=}(\cdot \bar{\varphi}, \bar{\varphi} \cdot): \mathcal{K}^{1 \times n} \multimap \mathcal{K}^{p \times 1}$ is a Galois connection.

Furthermore, the notion of a left (resp. right) reflexive, $(\mathcal{K}, \varphi)$, semiring is introduced in [5] as a complete idempotent semiring such that $(\langle\cdot \mid \cdot\rangle: K \times K \rightarrow K, \varphi)$ with $\langle\lambda \mid \mu\rangle=\lambda \mu$ induces a perfect Galois connection under the above-mentioned construction, that is for all $\lambda \in K,{ }^{-}\left(\lambda^{-}\right)=\lambda$ (resp. $\left.\left({ }^{-} \lambda\right)^{-}=\lambda\right)$. For the Boolean semiring we must choose $\varphi=0_{\mathcal{B}}$, the bottom in the order. For other semirings any invertible element may be chosen, e.g. $\varphi=e_{\mathcal{K}}$.

Note that $\varphi$ need not be unique: if $(\mathcal{K}, \varphi)$ is right (or left) reflexive, for any $\lambda \in K$ invertible, $(\mathcal{K}, \varphi \lambda)$ is left reflexive (and $(\mathcal{K}, \lambda \varphi)$ is right reflexive.) Finally, Cohen et al. [5] prove that idempotent semifields are left and right reflexive.

\section{$3 \quad \mathcal{K}$-Formal Concept Analysis}

We model $(\mathcal{K}$-valued $)$ sets of objects, $x \in \mathcal{X} \cong \mathcal{K}^{1 \times n}$, with row vectors in a left $\mathcal{K}$-semimodule and sets of attributes, $y \in \mathcal{Y} \cong \mathcal{K}^{p \times 1}$, with column vectors in a right $\mathcal{K}$-semimodule, as generalisations of characteristic functions in the powersets $\mathbf{2}^{G}, \mathbf{2}^{M}$, respectively ${ }^{5}$.

Definition 7 ( $\mathcal{K}$-valued formal context). Given two finite set of objects $G$ and attributes $M$, where $|G|=n$ and $|M|=p$, an idempotent semiring, $\mathcal{K}$, and a $\mathcal{K}$-valued incidence between them, $R \in \mathcal{K}^{n \times p}$, where $R(g, m)=\lambda$ reads as "object $g$ has attribute $m$ in degree $\lambda$ " and dually "attribute $m$ is manifested in object $g$ to degree $\lambda$ ", the triple $(G, M, R)_{\mathcal{K}}$ is called a $\mathcal{K}$-valued formal context.

Clearly the context can be represented as a $\mathcal{K}$-valued table with the value $R(g, m)=\lambda$ in the crossing of row $g$ with column $m$. Also, we are forced to admit that objects are isomorphic to elements of the space $\mathcal{K}^{1 \times p}$, that is rows of $R$ or object descriptions, vectors of as many values as attributes and attributes are isomorphic to elements of the space $\mathcal{K}^{n \times 1}$, columns of $R$ or attribute descriptions.

Proposition 1. Let $(\mathcal{K}, \varphi)$ be a reflexive, idempotent semiring. For a $\mathcal{K}$-valued formal context $(G, M, R)_{\mathcal{K}}$, with finite $|G|=n$ and $|M|=p$, there is at least one Galois connection between the power-sets of $\mathcal{K}$-valued sets of objects $\mathcal{K}^{1 \times n}$ and attributes $\mathcal{K}^{p \times 1}$

\footnotetext{
${ }^{5}$ This section follows in the tracks of $\S 1.1$ of [9].
} 
Proof. Recall that $\mathcal{X}=\mathcal{K}^{1 \times n}$ is a left semimodule and $\mathcal{Y}=\mathcal{K}^{p \times 1}$ a right semimodule, whence $\mathcal{X}^{o p}$ and $\mathcal{Y}^{o p}$ are right and left semimodules, respectively, whose multiplications are $R \stackrel{o p}{\longleftarrow} x=x^{t} \backslash R$ and $y \stackrel{o p}{\rightarrow} R=R / y^{t}$. We build a new bracket over the opposite semiring $\mathcal{K}^{o p}$ as given by $\langle y \mid x\rangle_{R}=y \stackrel{o p}{\rightarrow} R \stackrel{o p}{\longleftarrow} x=y^{t} \backslash R / x^{t}$ which is formally identical to the bracket over a relation in example 2 . Therefore, by the construction of section $2.3^{6}$ the maps:

$$
y_{\varphi}^{-}=\bigvee\left\{x \in X \mid\langle y \mid x\rangle_{R} \stackrel{o p}{\leq} \varphi\right\} \quad \bar{\varphi}^{-} x=\bigvee\left\{y \in Y \mid\langle y \mid x\rangle_{R} \stackrel{o p}{\leq} \varphi\right\}
$$

form a Galois connection $(\cdot \bar{\varphi}, \bar{\varphi} \cdot): \mathcal{Y}^{o p} \multimap \mathcal{X}^{o p}$. As requested in that section 2.3 , for $\mathcal{B}$ we must choose $\varphi=\epsilon_{\mathcal{B}}=\mathbf{0}$, but we are operating now in the opposite semiring $\mathcal{B}^{o p}$, hence we choose the bottom thereof, $\varphi=\epsilon_{\mathcal{B}^{o p}}=\mathbf{1}$. For any other semiring we may choose $\varphi=e_{\mathcal{K}}$.

Note that in an idempotent semifield we are guaranteed enough $\varphi$ to build as many connections: choose any invertible $\lambda \in K$, so that $\varphi=\lambda \otimes e_{\mathcal{K}}$.

Definition 8 ( $\varphi$-polars). Given a reflexive, idempotent semiring $(\mathcal{K}, \varphi)$ and a $\mathcal{K}$-valued formal context $(G, M, R)_{\mathcal{K}}$ satisfying the conditions of proposition 1 , we call $\varphi$-polars the dually adjoint maps of the corresponding Galois connection

$$
\begin{aligned}
& y_{\varphi}^{-}=\bigvee\left\{x \in X \mid\langle y \mid x\rangle_{R} \stackrel{o p}{\leq} \varphi\right\}=\bigwedge\left\{x \in X \mid\langle y \mid x\rangle_{R} \geq \varphi\right\}=(y \stackrel{o p}{\rightarrow} R) \backslash \varphi \\
& \bar{\varphi} x=\bigvee\left\{y \in Y \mid\langle y \mid x\rangle_{R} \stackrel{o p}{\leq} \varphi\right\}=\bigwedge\left\{y \in Y \mid\langle y \mid x\rangle_{R} \geq \varphi\right\}=\varphi \stackrel{o p}{/}(R \stackrel{o p}{\leftarrow} x)
\end{aligned}
$$

However, in this dualised construction the pivot describes a minimum degree of existence required for pairs $(x, y) \in X \times Y$ to be considered for operation.

Definition 9 (Formal $\varphi$-Concepts and $\varphi$-concept lattices). Given a reflexive, idempotent semiring $(\mathcal{K}, \varphi)$, a $\mathcal{K}$-valued formal context $(G, M, R)_{\mathcal{K}}$ with finite $|G|=n$ and $|M|=p$ and $\mathcal{K}$-valued vector spaces of rows $\mathcal{X} \cong \mathcal{K}^{1 \times n}$ and columns $\mathcal{Y} \cong \mathcal{K}^{p \times 1}$

1. A (formal) $\varphi$-concept of the formal context $(G, M, R)_{\mathcal{K}}$ is a pair $(a, b) \in$ $\mathcal{X} \times \mathcal{Y}$ such that ${ }_{\varphi}^{-} a=b$ and $b_{\varphi}^{-}=a$. We call $a$ the extent and $b$ the intent of the concept $(a, b)$, and $\varphi$ its (minimum) degree of existence.

2. If $\left(a_{1}, b_{1}\right)\left(a_{2}, b_{2}\right)$ are $\varphi$-concepts of a context, they are ordered by the relation $\left(a_{1}, b_{1}\right) \leq\left(a_{2}, b_{2}\right) \Longleftrightarrow a_{1} \leq a_{2} \Longleftrightarrow b_{1} \stackrel{o p}{\leq} b_{2}$, called the hierarchical order. The set of all concepts ordered in this way is called the $\varphi$-concept lattice, $\underline{\mathfrak{B}}_{\varphi}(G, M, R)_{\mathcal{K}}$, of the $\mathcal{K}$-valued context $(G, M, R)_{\mathcal{K}}$

Of course, the structure for the latter definition is proved next.

\footnotetext{
${ }^{6}$ And the demonstration of proposition $\S 24$ of [5]
} 
Theorem 2 (Fundamental theorem of $\mathcal{K}$-valued Formal Concept Analysis, finite version, $1^{\text {st }}$ half). Given a reflexive, idempotent semiring $(\mathcal{K}, \varphi)$, the $\varphi$-concept lattice $\mathfrak{B}_{\varphi}(G, M, R)_{\mathcal{K}}$ of a $\mathcal{K}$-valued formal context $(G, M, R)_{\mathcal{K}}$ with finite $|G|=n$ and $|M|=p$ is a (finite, complete) lattice in which infimum and supremum are given by:

$$
\bigwedge_{t \in T}\left(a_{t}, b_{t}\right)=\left(\bigoplus_{t \in T}^{o p} a_{t}, \bigoplus_{\varphi}^{-\mathrm{op}} a_{t \in \mathrm{T}} a_{t}\right) \bigvee_{t \in T}\left(a_{t}, b_{t}\right)=\left(\left[\bigoplus_{\mathrm{t} \in \mathrm{T}}^{\mathrm{op}} b_{t}\right]_{\varphi}^{-}, \bigoplus_{t \in T}^{o p} b_{t}\right)
$$

Proof. Recall that $a_{t} \in \mathcal{X}$ and $b_{t} \in \mathcal{Y}$ with $\mathcal{X} \cong \mathcal{K}^{1 \times n}$ and $\mathcal{Y} \cong \mathcal{K}^{p \times 1}$. The two dually isomorphic lattices $\bar{Y}$ and $\bar{X}$ are join semilattices of their ambient spaces, $\bar{Y} \subseteq \mathcal{X}^{o p}$ and $\bar{X} \subseteq \mathcal{Y}^{o p}$.

$$
\bar{Y}=\{\varphi \stackrel{o p}{\varphi}(R \stackrel{o p}{\longleftarrow} x) \mid x \in X\} \quad \bar{X}=\{(y \stackrel{o p}{\rightarrow} R) \stackrel{o p}{\backslash} \varphi \mid y \in Y\}
$$

Therefore by the inversion of the orders in opposite semimodules they are meet semilattices of $\mathcal{X}$ and $\mathcal{Y}$ respectively, hence the meets for $a_{t} \in \mathcal{X}$ and $b_{t} \in \mathcal{Y}$, and their $\varphi$-polars obtain the missing part of the concept.

Standard Formal Concept Analysis: an Example. At the end of section 1 we proposed a hypothesis about the origin of standard Formal Concept Analysis, for which we now provide the following corollary and informal proof. Of course, the wealth of results of Standard Formal Concept Analysis will not be available, specially those involving the second half of the Main Theorem, here missing.

Corollary 3. Standard Formal Concept Analysis is the concrete case of $\mathcal{K}$ valued $F C A$ where $\mathcal{K}$ is the Boolean semiring.

Proof (Informal). Recall that for the construction of proposition 1 the recommendation was to choose in the dualised semiring $\varphi=\epsilon_{\mathbb{B}^{o p}}=\mathbf{1}$ as pivot. In such case, we obtain $\mathfrak{B}(G, M, I)=\underline{\mathfrak{B}}_{1}(G, M, R)_{\mathbb{B}}$ and most of the basic results in Formal Concept Analysis follow from definitions 7-9 and theorem 2.

We now turn to an example about calculating extents, intents and concepts of a $\underline{\mathfrak{B}}_{1}(G, M, I)_{\mathbb{B}}$ lattice. For this purpose, we will use the context of [9], fig. 1.5 and associated concept lattice reproduced in figure 3 .

We represent the context in figure 4 as a matrix having 1 in the places occupied by crosses and $\mathbf{0}$ in the rest. In the same figure, we list the object and attribute concepts of the context adapted to our notation. Note that we introduce the singletons generating the concepts as row and column vectors, that is for objects, $\tilde{\gamma}(5)=\tilde{\gamma}([00001])$, and for attributes $\tilde{\mu}(c)=\tilde{\mu}\left([0010]^{t}\right)$.

To illustrate the calculations involved in the concept of object \#5 in the right hand side of the lattice with sets of objects and sets of attributes, we refer to table 1. In the first three columns of the table, we show, respectively, the extent of each concept, its left product by the matrix, the result of the whole bracket with the 


\begin{tabular}{|l||c|c|c|c|}
\hline & $\mathrm{a}$ & $\mathrm{b}$ & $\mathrm{c}$ & $\mathrm{d}$ \\
\hline \hline 1 & $\times$ & $\times$ & $\times$ & $\times$ \\
\hline 2 & $\times$ & $\times$ & & \\
\hline 3 & & $\times$ & $\times$ & $\times$ \\
\hline 4 & & $\times$ & & \\
\hline 5 & & $\times$ & $\times$ & \\
\hline
\end{tabular}

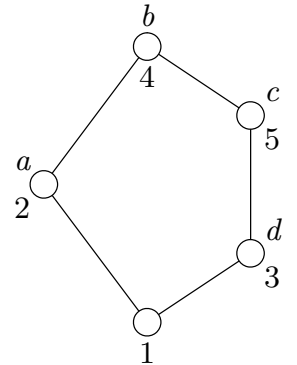

Fig. 3. The context and its concept lattice in [9] figs. $\S 1.5$ and 1.6.

$$
I=\left[\begin{array}{llll}
1 & 1 & 1 & 1 \\
1 & 1 & 0 & 0 \\
0 & 1 & 1 & 1 \\
0 & 1 & 0 & 0 \\
0 & 1 & 1 & 0
\end{array}\right] \quad \begin{aligned}
\tilde{\gamma}([10000]) & =\left([10000],[1111]^{t}\right) \\
\tilde{\gamma}([01000]) & =\tilde{\mu}\left([1000]^{t}\right)=\left([11000],[1100]^{t}\right) \\
\tilde{\gamma}([00100]) & =\tilde{\mu}\left([0001]^{t}\right)=\left([10100],[0111]^{t}\right) \\
\tilde{\gamma}([00010]) & =\tilde{\mu}\left([0100]^{t}\right)=\left([11111],[0100]^{t}\right) \\
\tilde{\gamma}([00001]) & =\tilde{\mu}\left([0010]^{t}\right)=\left([10101],[0110]^{t}\right)
\end{aligned}
$$

Fig. 4. The context as a Boolean matrix and its object and attribute concepts.

Table 1. Table showing the calculations described in the text

\begin{tabular}{|c|c|c|c|c|c|c|c|}
\hline$x_{i}$ & $R^{o p} \cdot x$ & ${ }_{5}\left|x_{i}\right\rangle$ & $\left|\left\langle y_{5} \mid x_{i}\right\rangle \stackrel{o p}{\leq} \varphi ?\right|$ & $y_{i}$ & $y_{i}{ }^{o p} I$ & $\left\langle y_{i} \mid x_{5}\right\rangle$ & $\mid\left\langle y_{i} \mid x_{5}\right\rangle \stackrel{o p}{\leq} \varphi ?$ \\
\hline$[10000]$ & [1111] & 1 & Yes & {$[1111]^{t}$} & {$[10000]^{t}$} & 0 & No \\
\hline$[11000]$ & [1100] & 0 & No & {$[1100]^{t}$} & {$[11000]^{t}$} & $\mathbf{0}$ & No \\
\hline$[10100]$ & [0111] & 1 & Yes & {$[0111]^{t}$} & {$[10100]^{t}$} & $\mathbf{0}$ & No \\
\hline [11111] & [0100] & 0 & No & {$[0100]^{t}$} & {$[11111]^{t}$} & 1 & Yes \\
\hline [10101] & [0110] & 1 & Yes & {$[0110]^{t}$} & {$[10101]^{t}$} & 1 & Yes \\
\hline
\end{tabular}


intent $y_{5}$ and whether this product complies with the restriction $\left\langle y_{5} \mid x_{i}\right\rangle \geq \mathbf{1}$, for the actual intent $y_{5}$. In the next four columns, the same operations are done based in the intents for the extent $x_{5}$.

Considering the lattice of extents, we see that the extents of the concepts that comply with the restriction $\left\langle y_{5} \mid x_{i}\right\rangle \geq \mathbf{1}$ are $\operatorname{ext}(\tilde{\gamma})(1)=[10000], \operatorname{ext}(\tilde{\gamma}(3))=$ [10100] and $\operatorname{ext}(\tilde{\gamma}(5))=[10101]$. Of these, $\operatorname{ext}(\tilde{\gamma}(5))$ is the minimum (in the opposite order.) Likewise, for intents complying with the restriction $\left\langle y_{i} \mid x_{5}\right\rangle \geq \mathbf{1}$, that is $\operatorname{int}(\tilde{\gamma}(4))=[0100]$ and $\operatorname{int}(\tilde{\gamma}(5))=[0110]$, the latter is the minimum $($ in the opposite order.)

\section{Discussion and Conclusions}

At the beginning of this paper, we started with a number of constraints and requirements for our endeavour. Where do they stand now?

Linear algebra and $\mathcal{K}$-Formal Concept Analysis. In section 1 we introduced a number of problems of interest in data mining. Specifically, recall problem 2 (adapted): for a set of objects $a$, find all other sets $a^{\prime}$ such that $R \stackrel{o p}{\leftarrow} a=R \stackrel{o p}{\leftarrow} a^{\prime}$. After the results in section 3, this amounts to finding the quotient class of the set of objects $a$ by the polar, $a(\bmod \bar{\varphi} \cdot)$; and similarly for the set of attributes $b$ finding the class, $b\left(\bmod \cdot \frac{-}{\varphi}\right)$. Note that problem 3 is essentially finding the quotient spaces without finding representatives for the classes.

As to the importance of such procedures for standard Formal Concept Analysis, a related procedure involving the closure maps is invoked in [9], §1.5 as an alternative to the standard reduction procedure based in arrow relations: find the kernels of the polars and form the quotient sets on objects and attributes modulo these kernels; the reduced incidence is actually the incidence between the corresponding classes in the quotient sets. We are confident that our results will help develop this alternative to context reduction.

Conclusions. We have tried to introduce in this paper a linear-algebraic perspective into Formal Concept Analysis whereby contexts may actually be represented as matrices, and the basic operations as multiplications in adequate algebras. And this with the twofold intention of bringing some light into the relation of logical operators and Formal Concept Analysis, and making the latter better adapted to deal with a broader class of quantitative problems.

These algebras happen to be a special kind of semirings, reflexive idempotent ones, and we have provided a construction for $\mathcal{K}$-Formal Concept Analysis generalising the standard analysis to allow for semiring-valued incidences. These results are not really surprising, since semirings seem to be closely related to Baer semigroups [3]. We still wonder whether idempotent semirings, will not actually be a sufficiently rich algebra to allow the kind of processing we put forward here. 
One instance of the above structure is the Boolean semiring and its opposite semimodule. We provide and example how standard Formal Concept Analysis seems to be the particularisation of our tehcnique for these semirings. Of course, the demonstration of the technique for actual data mining is still missing and will be the object of future papers.

\section{References}

1. F. Baccelli, G. Cohen, G. Olsder, and J. Quadrat. Synchronization and Linearity. Wiley, 1992.

2. R. Belohlávek. Lattices generated by binary fuzzy relations. In Int. Conf. on Fuzzy Set Theory and Applications, Slovakia, 1998.

3. T. Blyth and M. Janowitz. Residuation Theory. Pergamon press, 1972.

4. A. Burusco and R. Fuentes-González. The study of the l-fuzzy concept lattice. Mathware and Soft Computing, 1(3):209-218, 1994.

5. G. Cohen, S. Gaubert, and J.-P. Quadrat. Duality and separation theorems in idempotent semimodules. Linear Algebra and Its Applications, 379:395-422, 2004.

6. B. Davey and H. Priestley. Introduction to lattices and order. Cambridge University Press, Cambridge, UK, 2nd edition, 2002.

7. K. Denecke, M. Erné, and S. Wismath, editors. Galois Connections and Applications. Number 565 in Mathematics and Its Applications. Kluwer Academic, Dordrecht, Boston and London, 2004.

8. M. Erné. Adjunctions and Galois connections: Origins, History and Development, pages 1-138. In Denecke et al. [7], 2004.

9. B. Ganter and R. Wille. Formal Concept Analysis: Mathematical Foundations. Springer, Berlin, Heidelberg, 1999.

10. S. Gaubert. Two lectures on max-plus algebra. Support de cours de la 26-iéme École de Printemps d'Informatique Théorique, May 1998. http://amadeus.inria.fr/gaubert/papers.html.

11. S. Gaubert and the Maxplus Group. Methods and applications of $(\max ,+)$ linear algebra. Technical Report 3088, INRIA -, 1997.

12. G. Gediga and I. Dütsch. Approximation operators in qualitative data analysis. Technical Report CS-03-01, Department of Computer Science, Brock University, St. Catharines, Ontario, Canada, May 2003.

13. J. S. Golan. Power Algebras over Semirings. With Applications in Mathematics and Computer Science, volume 488 of Mathematics and its applications. Kluwer Academic, Dordrecht, Boston, London, 1999.

14. J. S. Golan. Semirings and Their Applications. Kluwer Academic, 1999.

15. E. Wagneur. Moduloïds and pseudomodules 1. dimension theory. Discrete Mathematics, 98:57-73, 1991.

16. R. Wille. Dyadic Mathematics - Abstractions from Logical Thought, pages 453-498. In Denecke et al. [7], 2004. 